\title{
Current status and future prospects of PARP inhibitor clinical trials in ovarian cancer
}

This article was published in the following Dove Press journal:

Cancer Management and Research

\section{Xuan Jiang \\ Weihua Li \\ Xiaoying $\mathrm{Li}$ \\ Huimin Bai \\ Zhenyu Zhang}

Department of Obstetrics and Gynecology, Beijing Chao-Yang Hospital,

Capital Medical University, Beijing,

People's Republic of China
Correspondence: Huimin Bai; Zhenyu

Zhang

Department of Obstetrics and

Gynecology, Beijing Chao-Yang Hospital,

Capital Medical University, No. 8, South

Road of Workers Stadium, Chaoyang

District, Beijing 100020, People's Republic

of China

Tel/Fax +86 I08523 I763

Email bhmdoctor@sina.com;

zhangzhylI@I63.com

\begin{abstract}
Poly (ADP-ribose) polymerase (PARP) inhibitors are a class of targeted agents for the treatment of solid tumors. Concurrent PARP inhibition in Breast Cancer Susceptibility Gene (BRCA)-mutated or homologous recombination-deficient tumor cells can induce "synthetic lethality", which targets two DNA repair pathways and induces serious cytotoxicity to tumor cells without damaging normal cells. Currently, PARP inhibitors such as olaparib, rucaparib and niraparib, which improve progression-free survival, particularly in patients harboring BRCA mutations, are approved by the Food and Drug Administration (FDA) and European Medicine Agency (EMA) for the treatment of ovarian cancers. Based on the results of different clinical trials, the indications for these drugs are slightly different. PARP inhibitors have been studied both as single agents and in combination with chemotherapy, antiangiogenic agents, and ionizing radiation. This review summarizes the critical clinical trials of PARP inhibitors that have been completed, provides an overview of the ongoing trials, presents the confirmed conclusions and notes the issues that need to be addressed in future studies.
\end{abstract}

Keywords: PARP inhibitor, BRCA mutation, olaparib, rucaparib, niraparib, ovarian cancer

\section{Introduction}

Epithelial ovarian cancer (EOC) is the leading cause of gynecologic cancer-related deaths in women. Most patients present with advanced-stage disease. ${ }^{1}$ Primary cytoreductive (debulking) surgery followed by platinum-based chemotherapy with or without concurrent and maintenance bevacizumab represents the currently recommended standard first-line systemic treatment for EOC, although most patients develop recurrence with a median progression-free survival (PFS) time of 12-18 months. Furthermore, the treatment efficacy diminishes over time and with chemotherapy cycles, and the toxicity of platinum drugs is cumulative. Moreover, the 5-year survival rate remains approximately $35 \% .^{2-5}$ Thus, more efficient treatment methods are warranted to improve the survival of ovarian cancer patients.

PARP inhibitors are oral small molecule inhibitors of poly (ADP-ribose) polymerase (PARP) enzymes 1, 2 and 3 and have recently demonstrated great clinical efficacy among ovarian cancer patients. PARP inhibitors are the first FDA-approved biological agent for ovarian cancer based on the individualized features of cancer. ${ }^{6}$ Patients with BRCA1/2-mutated or homologous recombination-deficient (HRD) ovarian tumors can benefit from PARP inhibitors. Currently, PARP inhibitors such as olaparib, rucaparib and niraparib have been approved by the FDA and EMA for the treatment of ovarian cancer. ${ }^{7-9}$ The indications for PARP inhibitors approved in Europe (EU) and the United States (US), such as olaparib capsules, 
olaparib tablets, rucaparib, and niraparib, are listed in Table 1. Other PARP inhibitors, such as veliparib and talazoparib, are in various stages of clinical development. ${ }^{6}$ A PubMed literature search was conducted using the search term "PARP inhibitors and ovarian cancer". The ClinicalTrials.gov website was searched to identify relevant clinical trials evaluating PARP inhibitors in ovarian cancer. An on-line search was conducted to identify all FDA approvals in the US and EMA approvals in EU of PARP inhibitors for ovarian cancer treatment. Proceedings from scientific meetings were searched to identify abstracts reporting unpublished data from clinical trials. This review summarizes the critical clinical trials on PARP inhibitors and augments our understanding of the past, present and future of PARP inhibitors.

\section{Rationale for the mechanism of action of PARP inhibitors}

The family of PARP enzymes ( $\geq 17$ enzymes) is primarily involved in detecting single-strand breaks (SSBs) and triggering a cascade of events leading to the recruitment of DNA repair factors. PARP1, acting as a sensor and signal transducer for SSBs, binds DNA and catalyzes a series of PARylation events leading to PARP1 autoPARylation, which in turn induces the release of PARP1 from DNA. PARP inhibitors prevent the release of PARP1 from DNA by inhibiting autoPARylation, resulting in persistent SSBs, stalled replication forks and subsequently, double-strand breaks (DSBs). ${ }^{8,10-12}$ The repair of DSBs relies on two pathways: homologous recombination (HR) and nonhomologous end-joining (NHEJ). ${ }^{11,13} \mathrm{HR}$ is a high-fidelity, while NHEJ is an error-prone, low-fidelity repair system, which directly ligates the ends of a DSB, resulting in the deletion or mutation of DNA sequences, genomic instability, cell cycle arrest, and cell apoptosis. ${ }^{10,11,13}$ BRCA1 and BRCA2 are crucial proteins involved in mediating the HR repair pathway. Therefore, concurrent inhibition of PARP enzymes with BRCA1 or BRCA2 deficiency is synthetically lethal to tumor cells because the loss of function of either alone is compatible with cell viability, but the concurrent loss of both results in cell death. ${ }^{14,15}$

Of note, except for the catalytic inhibition of PARP1 autoPARylation, PARP inhibitors promote the trapping of PARP1-DNA complexes, and the cytotoxicity of the PARP inhibitors mainly depends on their trapping potential. All PARP inhibitors inhibit PARP1 and PARP2 in vitro at nanomolar concentrations but exhibit different levels of PARP-trapping ability on the DNA SSB sites.
Talazoparib exhibits the highest potency in trapping PARPs and the highest single-agent cytotoxicity, 100-fold higher than that of olaparib and rucaparib; niraparib exhibits higher potency in trapping PARPs than olaparib and rucaparib, and the PARP-trapping ability of veliparib is the least potent. Therefore, the forming of PARP1-DNA complexes is crucial for the trapping ability of PARP inhibitors. PARP inhibition is not equivalent to PARP deletion. In vitro studies have demonstrated that PARP inhibitors have no effect on tumor cells with the complete absence of the PARP1 enzyme. ${ }^{16}$ Table 1 shows the dissociation constant $(\mathrm{Ki})$ reflecting the catalytic inhibition of PARP1 and the PARP-trapping capacity of PARP inhibitors (olaparib, rucaparib, niraparib, veliparib and talazoparib).

\section{Approval indications of three PARP inhibitors in ovarian cancer}

In December 2014, the olaparib capsule was approved in the EU as a maintenance monotherapy for adult patients with platinum-sensitive, recurrent BRCA1/2-mutated (germline and/or somatic, g/s) high-grade serous epithelial ovarian, fallopian tube or primary peritoneal cancer (collectively termed ovarian cancer) (HGSOC) who have demonstrated complete or partial response $(\mathrm{CR} / \mathrm{PR})$ to platinum-based chemotherapy. ${ }^{17}$ At the same time, the olaparib capsule was also approved in the US as monotherapy for patients with germline BRCA mutation (gBRCAm) and advanced ovarian cancer treated with three or more prior lines of chemotherapy. ${ }^{18}$ In August 2017, an olaparib tablet was approved in the US as a maintenance monotherapy for adult patients with recurrent ovarian cancer who have demonstrated CR/PR to platinum-based chemotherapy and for the treatment of adult patients with gBRCAm and advanced ovarian cancer treated with three or more prior lines of chemotherapy. ${ }^{19}$ In February 2018, the olaparib tablet was approved in the EU as a maintenance monotherapy for adult patients with platinum-sensitive recurrent high-grade ovarian carcinoma (HGOC) who have demonstrated $\mathrm{CR} / \mathrm{PR}$ to platinumbased chemotherapy. ${ }^{20}$ In December 2018, an olaparib tablet was approved in the US as a first-line maintenance treatment in BRCA1/2-mutated, advanced ovarian cancer after a CR/PR to first-line platinum-based chemotherapy. ${ }^{21}$

In December 2016, rucaparib was approved in the US for the treatment of adult patients with $\mathrm{g} / \mathrm{sBRCA}$ mutation-associated ovarian cancer treated with two or more prior lines of chemotherapy and as a maintenance monotherapy for adult patients with recurrent 
Table I Approval indications, dosing, dissociation constant $(\mathrm{Ki})$, and relative trapping capacity of PARP inhibitors ${ }^{17-25}$

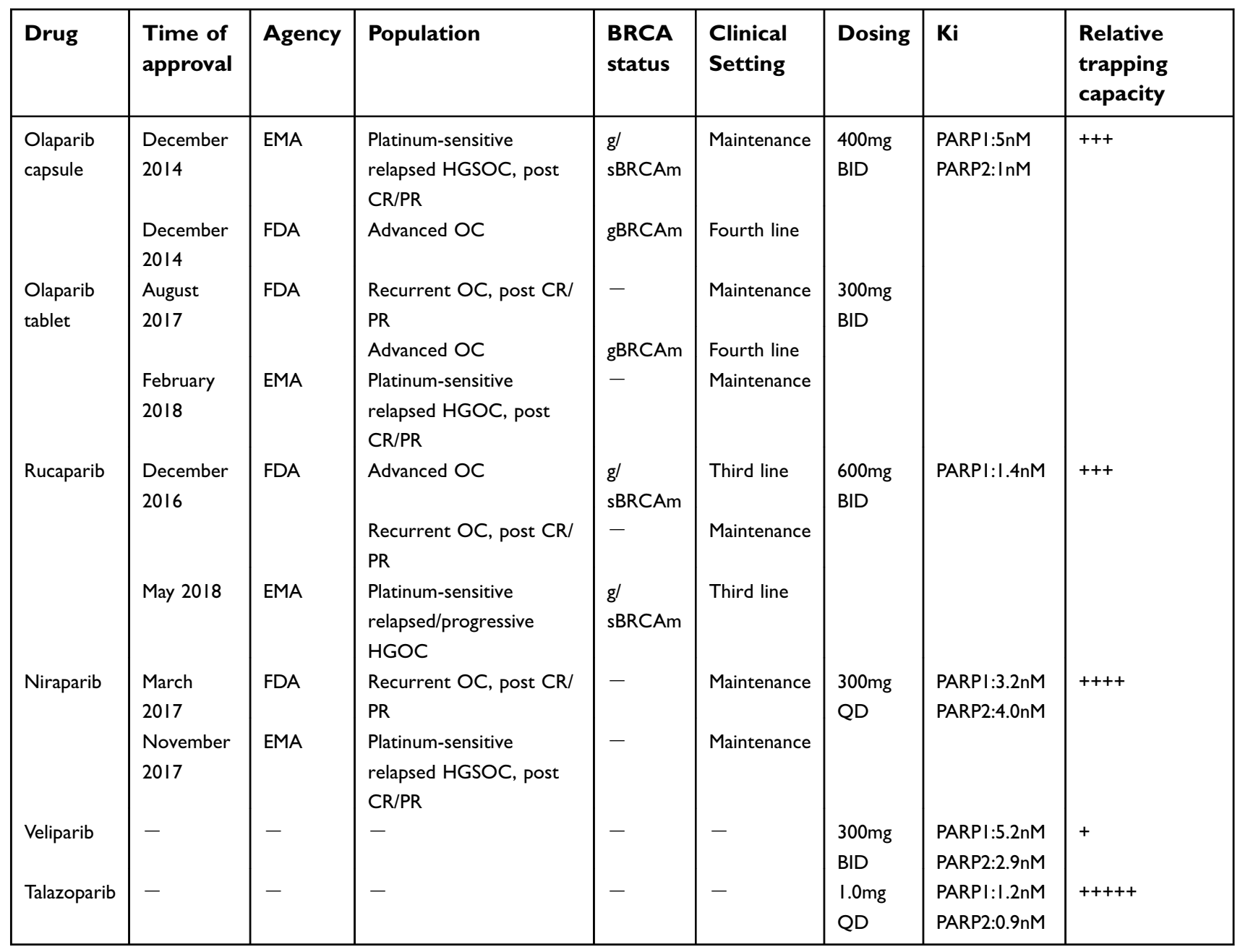

Note: The relative trapping capacity of the PARP inhibitors is talazoparib (+++++) $\square$ niraparib (++++) $\square$ olaparib (+++) and rucaparib (+++) $\square$ veliparib (+).

Abbreviations: FDA, Food and Drug Administration; EMA, European Medicine Agency; BRCA, breast cancer susceptibility gene; PARP, poly (ADP-ribose) polymerase; CR/ $\mathrm{PR}$, complete response or partial response; g/sBRCAm, germline and/or somatic BRCAI/2 mutation; OC, epithelial ovarian, fallopian tube or primary peritoneal cancer; HGOC, High-grade epithelial ovarian, fallopian tube or primary peritoneal cancer; HGSOC, High-grade serous epithelial ovarian, fallopian tube or primary peritoneal cancer; QD, once daily; BID, twice daily.

ovarian cancer who have demonstrated $\mathrm{CR} / \mathrm{PR}$ to platinum-based chemotherapy. ${ }^{22}$ In May 2018, rucaparib was approved in the EU for the treatment of adult patients with platinum-sensitive, relapsed or progressive, g/sBRCA1/2-mutated HGOC treated with two or more prior lines of platinum-based chemotherapy. ${ }^{23}$

In March 2017, niraparib was approved in the US as a maintenance monotherapy for adult patients with recurrent ovarian cancer who have demonstrated CR/ PR to platinum-based chemotherapy. ${ }^{24}$ In November 2017, niraparib was also approved in the EU as a maintenance monotherapy for adult patients with platinum-sensitive, relapsed, HGSOC who have demonstrated CR/PR to platinum-based chemotherapy. ${ }^{25}$
To summarize, in the EU, the three PARP inhibitors can be administered only to patients with platinum-sensitive, relapsed HGSOC. Additionally, rucaparib is indicated as the third-line treatment in BRCA1/2 mutation-associated ovarian cancer; however, olaparib and niraparib are indicated only as maintenance treatment. In the US, olaparib is indicated as the fourth-line treatment for BRCA1/2-mutated advanced ovarian cancer, the maintenance treatment for recurrent ovarian cancer and the first-line maintenance treatment for newly-diagnosed, BRCA1/2-mutated, advanced ovarian cancer; rucaparib is indicated as both the third-line treatment for BRCA1/2 mutation-associated ovarian cancer and the maintenance treatment for recurrent ovarian cancer; and niraparib is indicated only as the maintenance treatment for recurrent ovarian cancer. 


\section{Clinical development of PARP inhibitor efficacy in ovarian cancer}

The critical clinical trials of PARP inhibitors, including olaparib, rucaparib and niraparib, in ovarian cancer are summarized in Table 2.

\section{Olaparib}

Initially, the FDA approved olaparib as the fourth-line treatment for advanced ovarian cancer with gBRCAm, based on the results from Study 42, a phase II study demonstrating an objective response rate (ORR) of $31 \%$ and a median overall survival (OS) time of 16.6 months with olaparib treatment in 193 ovarian cancer patients (NCT01078662). Patients with platinum-resistant disease or those unsuited for further platinum therapy due to significant toxicity or hypersensitivity to platinum, were also included in this trial. This level of activity significantly exceeded that of conventional third-/fourth-line therapy; hence, the FDA approved olaparib for this indication.${ }^{26}$ A pooled analysis of 6 phase I/II trials [NCT00516373 (Study 2), NCT00777582 (Study 24), NCT00494442 (Study 9), NCT00628251 (Study 12), NCT00679783 (Study 20), and NCT01078662 (Study 42)] identified the ORR as 36\% and the median duration of response as 7.4 months with olaparib treatment among patients with gBRCAm and advanced relapsed ovarian cancer. The ORR among patients who had received three or more lines of prior chemotherapy was $31 \%$, with a duration of response of 7.8 months, indicating that a sustained response to olaparib could be achieved in heavily pretreated, relapsed, gBRCAm-associated ovarian cancers. ${ }^{27}$

The maximum tolerated dose (MTD) of olaparib was identified as $400 \mathrm{mg}$ twice daily in a phase I trial (NCT00516373). ${ }^{28}$ A dose-response relationship between different olaparib dose levels was studied in two phase II trials. In the first phase II study (NCT00628251), the ORR was observed to be higher in the $400 \mathrm{mg}$ olaparib group (31\%) than in the $200 \mathrm{mg}$ olaparib group (25\%). ${ }^{29}$ In another phase II study (NCT00494442), a difference of 3.9 months in the median PFS was observed in the $400 \mathrm{mg}$ olaparib group compared with the $100 \mathrm{mg}$ olaparib group, in favor of the $400 \mathrm{mg}$ olaparib group. And the ORRs were $33 \%$ and $13 \%$ in the $400 \mathrm{mg}$ olaparib and $100 \mathrm{mg}$ olaparib groups, respectively. ${ }^{30}$ However, neither of these two trials had sufficient power to address the efficacy difference between the two olaparib dose levels. Therefore, whether olaparib exhibits a dose-response relationship requires additional evaluation.

Study 19, SOLO2 and SOLO1 are all randomized, double-blind, placebo-controlled phase II/III studies of olaparib monotherapy that are highly significant (Study 19: NCT00753545, SOLO2: NCT01874353, and SOLO1: NCT01844986). Study 19 showed that olaparib maintenance monotherapy significantly improved PFS [median, 8.4 vs 4.8 months; hazard ratio (HR) $0.35,95 \%$ confidence interval (CI) $0.25-0.49 ; p<0.001]$ compared with placebo in patients with platinum-sensitive, recurrent HGSOC who had received 2 or more prior lines of platinum-based chemotherapy and demonstrated a CR/PR to the most recent platinum-based chemotherapy. ${ }^{31}$ Retrospective germline and somatic BRCA mutation testing was performed on all patients with an additional 2 years of follow-up. A total of $51 \%$ of the HGSOC population had a germline or somatic BRCA mutation, and patients with or without $\mathrm{g} / \mathrm{sBRCA}$ mutations both gained the PFS benefit from olaparib maintenance therapy versus placebo, with a greater PFS benefit in the $\mathrm{g} / \mathrm{sBRCA} 1 / 2$-mutated group than in the wild-type BRCA group ( $\mathrm{g} / \mathrm{sBRCA} 1 / 2$-mutated: 11.2 vs 4.3 months; HR $0.18,95 \%$ CI [0.10-0.31]; $p<0.0001$; wild-type BRCA: 7.4 vs 5.5 months; HR 0.54, 95\% CI [0.34-0.85]; $p=0.0075) .{ }^{32}$ The first, second and third interim OS analyses from Study 19 were performed after $38 \%, 58 \%$ and $77 \%$ of patients had died, respectively. The final OS analysis was performed after 210 deaths (79\% data maturity), after a median follow-up of 6.5 years. ${ }^{31-34}$ Neither of the first or second interim OS analyses showed a benefit for olaparib versus placebo for either the BRCA1/2-mutated or BRCA wild-type groups in the overall population. ${ }^{32}$ The third interim OS analysis and the final OS analysis both showed an OS advantage of olaparib versus placebo in all patients (median OS 29.8 vs 27.8 months, HR $0.73,95 \%$ CI $0.55-0.95, p=0.02)$ and in patients with BRCA mutations (34.9 vs 30.2 months, HR $0.62,95 \%$ CI $0.42-0.93, p=0.02) .{ }^{33}$ However, the predefined threshold for statistical significance $(p=0.0095)$ was not met. ${ }^{33,34}$ In the final OS analysis, 32 patients (24\%) had received olaparib maintenance for over 2 years, and 15 (11\%) had received olaparib maintenance for over 6 years, which demonstrated the long-term safety and tolerability of olaparib maintenance therapy. ${ }^{34}$

Therefore, olaparib maintenance significantly improved PFS in patients with platinum-sensitive recurrent HGSOC treated with two or more previous lines of platinum-based chemotherapy, and patients with a g/sBRCA mutation had the greatest benefit from olaparib. ${ }^{31,32}$ The analyses for time to first subsequent therapy or death, time to second progression, and time to second subsequent therapy or death showed that the PFS benefit was sustained until subsequent 







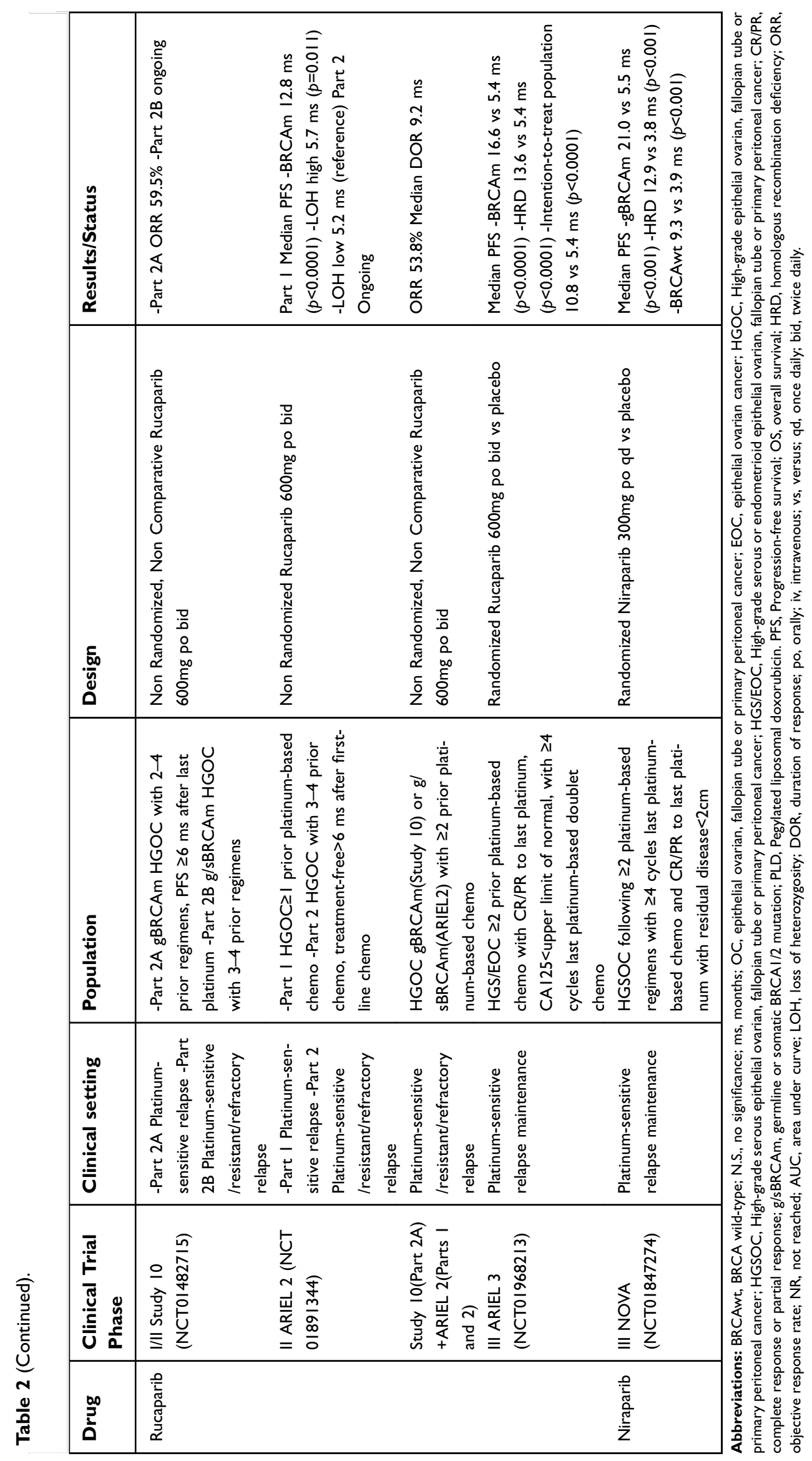


treatment and that a long-term benefit was achieved irrespective of BRCA1/2 mutation status. ${ }^{32,33}$ The long followup time required to obtain sufficient OS data increases the chance that post-progression PARP inhibitor therapy and patient crossover will effect the OS data. When excluding the patients from places where placebo patients were treated with post-progression PARP inhibitors, the OS hazard ratio was significantly improved, indicating that in Study 19, post-progression PARP inhibitor therapy had a confounding effect on the interim OS analysis for patients with BRCA mutations. $^{35}$

SOLO2 (NCT01874353) aimed to investigate the efficacy and safety of olaparib in platinum-sensitive, recurrent ovarian cancer patients with a $\mathrm{g} / \mathrm{sBRCA} 1 / 2$ mutation who had received two or more lines of previous chemotherapy and demonstrated a $\mathrm{CR} / \mathrm{PR}$ to the most recent platinumbased chemotherapy. The median PFS was significantly longer with olaparib (19.1 months) than with placebo (5.5 months; HR 0.30 [95\% CI 0.22-0.41], $p<0.0001$ ). The PFS benefit from olaparib maintenance compared with that from placebo in SOLO2 substantially exceeded that observed in Study 19, which is not surprising because SOLO2 included only patients with $\mathrm{g} / \mathrm{sBRCA} 1 / 2$-mutated tumors. $^{36}$ Furthermore, heavily pretreated patients with BRCA1/2-mutated ovarian cancer whose disease progressed following PARP inhibitor therapy retain the potential to respond to subsequent chemotherapy, including platinum-based chemotherapy. The ORR to subsequent chemotherapy and platinum-based chemotherapy was reported to be as high as $36 \%$ and $40 \%$, respectively. ${ }^{37,38}$

SOLO 1 (NCT01844986) aimed to evaluate the efficacy and safety of olaparib versus placebo in patients with BRCA1/2-mutated advanced (FIGO stage III-IV) highgrade serous or endometrioid ovarian cancer, primary peritoneal cancer or fallopian tube cancer (HGS/EOC) following $\mathrm{CR} / \mathrm{PR}$ to initial first-line platinum-based chemotherapy. SOLO 1 was the first trial to investigate the efficacy of olaparib as a first-line maintenance therapy for primary advanced ovarian cancers. Patients who had no evidence of disease at 2 years stopped receiving the trial intervention. Maintenance olaparib led to a substantial improvement in the PFS of patients with newly diagnosed advanced ovarian cancer and BRCA mutation, with a difference of approximately 3 years in the median PFS for olaparib compared with that of placebo (the median PFS in the placebo group was 13.8 months, and up to $53 \%$ of patients in the olaparib group had no recurrence after 48 months of follow-up). The Kaplan-Meier curves for the olaparib group did not appreciably change after 2 years, suggesting an enduring treatment benefit after treatment cessation. The second PFS showed a statistically significant improvement, suggesting that olaparib did not diminish patients' ability to benefit from subsequent therapy. ${ }^{39}$ Considering the significant PFS advantage in favor of firstline maintenance treatment with PARP inhibitors, patients would have to undergo g/sBRCA testing immediately after ovarian cancer diagnosis and adopt the PARP inhibitor first-line maintenance treatment if they were positive for g/sBRCA mutation. Moreover, the PFS benefit from maintenance olaparib compared with that from placebo in SOLO1 also substantially exceeded that in SOLO2, indicating that olaparib is more beneficial to BRCA mutation carriers as a first-line maintenance treatment than as a third-line treatment.

\section{Rucaparib}

An integrated analysis of two single-arm clinical trials (Study 10, NCT01482715; ARIEL2, NCT01891344)

Table 3 The safety profiles of PARP inhibitors (olaparib, rucaparib and niraparib) in clinical trials $27,31,36,39-43,45$

\begin{tabular}{|c|c|c|c|c|c|c|c|}
\hline Drugs & Study & $\begin{array}{l}\text { Grade 3/ } \\
4 \text { AEs }\end{array}$ & $\begin{array}{l}\text { Dose inter- } \\
\text { ruption }\end{array}$ & $\begin{array}{l}\text { Dose } \\
\text { reduction }\end{array}$ & $\begin{array}{l}\text { Dose disconti- } \\
\text { nuation }\end{array}$ & $\begin{array}{l}\text { MDS/ } \\
\text { AML }\end{array}$ & $\begin{array}{l}\text { Treatment } \\
\text { related deaths }\end{array}$ \\
\hline \multirow[t]{4}{*}{ Olaparib } & $\begin{array}{l}\text { Study } 2 / 24 / 9 / 12 / 20 / \\
42(n=223)\end{array}$ & $54 \%$ & $40 \%$ & $4 \%$ & $7 \%$ & $2 \%$ & $3.6 \%$ \\
\hline & Study $19(n=136)$ & $35.3 \%$ & $27.9 \%$ & $22.8 \%$ & $2.2 \%$ & $2 \%$ & $0 \%$ \\
\hline & SOLO2(n=195) & $36 \%$ & $45 \%$ & $25 \%$ & $11 \%$ & $2 \%$ & $1 \%$ \\
\hline & SOLOI (n=260) & $39 \%$ & $52 \%$ & $28 \%$ & $12 \%$ & $1 \%$ & $0 \%$ \\
\hline \multirow[t]{2}{*}{ Rucaparib } & $\begin{array}{l}\text { ARIEL2 + Study } 10 \\
(n=377)\end{array}$ & $60.7 \%$ & $58.6 \%$ & $45.9 \%$ & $10 \%$ & $0.5 \%$ & $0 \%$ \\
\hline & ARIEL3(n=372) & $56 \%$ & $64 \%$ & $55 \%$ & $13 \%$ & $1 \%$ & $1 \%$ \\
\hline Niraparib & $\operatorname{NOVA}(n=367)$ & $64.6 \%$ & $68.9 \%$ & $66.5 \%$ & $14.7 \%$ & $1.4 \%$ & $0.3 \%$ \\
\hline
\end{tabular}

Abbreviations: AEs, Adverse Events; MDS/AML, myelodysplastic syndrome/acute myeloid leukemia; PARP, poly (ADP-ribose) polymerase. 
Table 4 The adverse events rates of PARP inhibitors (olaparib, rucaparib and niraparib) in clinical trials ${ }^{27,31,36,39-43,45}$

\begin{tabular}{|c|c|c|c|c|c|c|c|}
\hline \multirow[t]{2}{*}{ Grade 3/4 AEs } & \multicolumn{4}{|l|}{ Olaparib } & \multicolumn{2}{|l|}{ Rucaparib } & \multirow{2}{*}{$\begin{array}{l}\text { Nirparib } \\
\text { NOVA } \\
(n=367)\end{array}$} \\
\hline & $\begin{array}{l}\text { Study } 2 / 24 / 9 / 12 / \\
20 / 42(n=223)\end{array}$ & $\begin{array}{l}\text { Study } 19 \\
(n=136)\end{array}$ & $\begin{array}{l}\text { SOLO2 } \\
(n=\mid 95)\end{array}$ & $\begin{array}{l}\text { SOLOI } \\
(n=260)\end{array}$ & $\begin{array}{l}\text { ARIEL2 + Study I0 } \\
(n=377)\end{array}$ & $\begin{array}{l}\text { ARIEL3 } \\
(n=372)\end{array}$ & \\
\hline Anemia & $15 \%$ & $5.1 \%$ & $19 \%$ & $22 \%$ & $24.9 \%$ & $19 \%$ & $25.3 \%$ \\
\hline Neutropenia & - & - & $5 \%$ & $9 \%$ & $9.8 \%$ & $7 \%$ & $19.6 \%$ \\
\hline Thrombocytopenia & - & - & $1 \%$ & $1 \%$ & $4.5 \%$ & $5 \%$ & $33.8 \%$ \\
\hline Fatigue/asthenia & $7 \%$ & $7.3 \%$ & $4 \%$ & $4 \%$ & $10.9 \%$ & $7 \%$ & $8.2 \%$ \\
\hline Nausea & $3 \%$ & $2.2 \%$ & $3 \%$ & $1 \%$ & $5.0 \%$ & $4 \%$ & $3.0 \%$ \\
\hline Vomiting & $4 \%$ & $2.2 \%$ & $3 \%$ & $\square 1 \%$ & $4.0 \%$ & $4 \%$ & $1.9 \%$ \\
\hline
\end{tabular}

Abbreviations: AEs, Adverse Events; PARP, poly (ADP-ribose) polymerase.

evaluated the efficacy and safety of rucaparib in 106 advanced ovarian cancer patients who had progressed after two or more prior lines of chemotherapy. ${ }^{40,41}$ The ORR was $54 \%$ in all patients and $66 \%, 25 \%$, and $0 \%$ in platinum-sensitive, platinum-resistant and platinum-refractory patients, respectively. ${ }^{42}$ The recommended dose of rucaparib was identified as $600 \mathrm{mg}$ twice daily in the phase I part of Study $10 .{ }^{40}$ The first part of ARIEL2 aimed to assess the ability of tumor genomic loss of heterozygosity ( $\mathrm{LOH}$ ) as a biomarker, beyond the g/ sBRCA1/2 mutation, to predict the response to rucaparib. Patients with platinum-sensitive recurrent HGS/EOC who had received one or more prior lines of platinum-based chemotherapy and had progressed 6 months or more after the most recent platinum-based chemotherapy were classified into one of three predefined HRD subgroups: g/ sBRCA mutant, BRCA wild-type and LOH high, and BRCA wild-type and LOH low. Compared with that of the LOH low subgroup (median 5.2 months), the PFS was significantly longer in the BRCA mutant (median 12.8 months, HR $0.27,95 \%$ CI $0.16-0.44, p<0.0001)$ and LOH high (5.7 months, $0.62,0.42-0.90, p=0.011$ ) subgroups. These results indicate that tumor genomic $\mathrm{LOH}$ can be used as a biomarker, beyond the g/sBRCA1/2 mutation, to identify patients with BRCA wild-type platinum-sensitive ovarian cancers who might benefit from rucaparib. $^{41}$ In a phase III trial (ARIEL3, NCT01968213), compared to placebo, rucaparib maintenance significantly improved PFS in patients with platinum-sensitive recurrent HGS/EOC treated with two or more prior lines of platinum-based chemotherapy and following $\mathrm{CR} / \mathrm{PR}$ to platinum-based chemotherapy, which included the BRCA1/2-mutated (16.6 vs 5.4 months, $p<0.0001$ ), HRD (13.6 vs 5.4 months, $p<0.0001$ ), and intention-to-treat populations (10.8 vs 5.4 months, $p<0.0001) .^{43}$

\section{Niraparib}

The MTD of niraparib was identified in a phase I dose escalation study as $300 \mathrm{mg}$ daily. ${ }^{44}$ The approval of niraparib was based on a randomized phase III trial (ENGOT-OV16/NOVA, NCT01847274), in which patients with platinum-sensitive, recurrent ovarian cancer and a CR/PR after two or more prior lines of platinum-based chemotherapy were treated with niraparib or placebo maintenance. Niraparib maintenance significantly improved the PFS, compared to placebo, irrespective of the gBRCAm or HRD status (21.0 vs 5.5 months in the gBRCAm group, 12.9 vs 3.8 months in the HRD plus BRCA wild-type group, and 9.3 vs 3.9 months in the overall BRCA wild-type group [ $p<0.001])$. Compared to placebo, niraparib also significantly improved the time to second progression and chemotherapy-free interval in the gBRCAm, BRCA wild-type, and HRD subgroups. Similar to tumor genomic LOH, HRD status as a biomarker might indicate the potential usefulness of PARP inhibitors; however, the absence of either does not preclude benefit from niraparib maintenance therapy. ${ }^{45}$

\section{Veliparib}

The MTD of veliparib was identified in a phase I/II study (NCT01472783) as $300 \mathrm{mg}$ twice daily. In this study, the ORR of veliparib monotherapy in patients with gBRCA1/2mutated, platinum-resistant or intermediate-sensitive (disease relapse within 6 to 12 months of previous platinum-based therapy) relapsed ovarian cancer was $65 \%$ (6\% CR and 59\% PR). The PFS and OS of the intention-to-treat population were 5.6 months and 13.7 months, respectively. Treatment with veliparib in heavily pretreated, recurrent ovarian cancer 
patients demonstrates considerable efficacy with an acceptable toxicity profile. ${ }^{46}$ A phase II trial (NCT01540565) studied the efficacy and tolerability of veliparib monotherapy (400 mg twice daily) in persistent or recurrent EOC patients with gBRCAm after three or fewer prior chemotherapy regimens. The ORRs were $26 \%, 20 \%$ and $35 \%$ for the overall, platinumresistant and platinum-sensitive patient populations, respectively. $^{47}$

\section{Talazoparib}

In a phase I dose-escalation study (NCT01286987), talazoparib demonstrated single-agent antitumor activity and was well tolerated at an MTD of $1.0 \mathrm{mg} /$ day. At $1.0 \mathrm{mg} /$ day, clinical responses were observed in 5 of 12 (42\%) patients with BRCA1/2-mutated ovarian cancers. ${ }^{48}$

\section{Safety profiles of PARP inhibitors in ovarian cancer}

The safety data for olaparib treatment in a pooled analysis of 6 phase I/II trials (Study 2, Study 24, Study 9, Study 12, Study 20, and Study 42), in Study 19, in SOLO2 and in SOLO1; for rucaparib treatment in ARIEL3 and in an integrated analysis of Study 10 and ARIEL2; and for niraparib treatment in NOVA are shown in Tables 3 and 4. The occurrence of treatment-related deaths was the highest (up to $3.6 \%$ ) in the pooled analysis of 6 phase I/ II trials; these deaths were attributed to acute leukemia, chronic obstructive pulmonary disease, pulmonary embolism, cerebrovascular accident, intestinal perforation, sepsis, and suture rupture. However, none of the adverse events (AEs) leading to death was considered causally related to olaparib. ${ }^{27}$ The secondary malignancies myelodysplastic syndrome/acute myeloid leukemia (MDS/AML) seemed to be prominent problems associated with PARP inhibitor treatment. The incidence of MDS/AML was approximately $2 \%$ in 298 patients with advanced cancer and a deleterious gBRCAm treated with olaparib in Study $42 ;{ }^{26}$ in 136 patients with advanced relapsed ovarian cancer treated with olaparib in Study $19 ;^{31}$ and in 195 patients with advanced g/sBRCA1/2-mutated, relapsed ovarian cancer treated with olaparib in SOLO2. ${ }^{36}$ However, confirming whether the secondary MDS/AML was causally related to PARP inhibitor treatment was difficult because the patients had undergone multiple lines of chemotherapy before they were enrolled in the trial, as proven by the high rate of secondary AML/MDS in patients in the placebo group, up to $4 \%$ in 99 patients with advanced ovarian cancer and a BRCA1/2 mutation, in $\mathrm{SOLO} 2 .{ }^{36}$ In addition, MDS/AML occurred in 5 of 367 (1.4\%) patients receiving niraparib and in 2 of 179 (1.1\%) patients receiving placebo in NOVA; ${ }^{45} \mathrm{MDS} / \mathrm{AML}$ were reported in three $(1 \%)$ patients in the rucaparib group but in no patients in the placebo group in ARIEL3. ${ }^{43}$ MDS/AML occurred in 3 of $260(1 \%)$ patients in the olaparib group but in none of the 130 patients in the placebo group in SOLO1, in which the patients had undergone only first-line platinum-based chemotherapy and olaparib maintenance therapy. ${ }^{39}$

The incidence of MDS/AML in a large case-control study of 28,971 ovarian cancer patients who had received prior platinum therapy between 1980 and 1993 was $0.33 \%{ }^{49}$ An epidemiological analysis identified 109 $(0.17 \%)$ therapy-related myeloid leukemia cases in 63,359 patients after treatment for EOC from 1973-2006. The development of secondary leukemia with EOC diagnosis was significantly decreased following a shift from a melphalan and platinum regimen to a paclitaxel and platinum regimen. ${ }^{50}$ The incidence of MDS/AML in a large cohort study of 23,862 ovarian cancer patients who had received DNA-damaging therapy between 2000 and 2014 was $0.8 \%$, and the duration of exposure to DNA-damaging therapy was demonstrated to be a significant risk factor for developing MDS/AML during follow-up. ${ }^{51}$ The incidence of $\mathrm{MDS} / \mathrm{AML}$ in a population of patients carrying a gBRCAm is unknown. Therefore, whether the high incidences of secondary MDS/AML in Study 42, Study 19 and SOLO2 were correlated with the high rates of BRCA mutation among these patients is also unknown. Based on these figures, secondary MDS/AML cannot be definitively regarded as causally related to PARP inhibitor treatment. However, patients should be warned of the risks and be monitored for hematologic toxicity. Further investigations are warranted.

The predominant AE with PARP inhibitor treatment is anemia. The incidence of grade $3 / 4$ anemia was reported to be as high as $22 \%$ in $\mathrm{SOLO}^{39}$ and $25 \%$ in ARIEL2 + Study $10^{42}$ and NOVA, ${ }^{45}$ far higher than the incidence of other AEs such as fatigue/asthenia, nausea, and vomiting. Hematologic toxicity was more serious with niraparib treatment than with olaparib or rucaparib treatment. Both thrombocytopenia (33.8\%) and neutropenia (19.6\%) were prominent AEs with niraparib treatment, ${ }^{45}$ compared with their incidence with olaparib treatment [thrombocytopenia $(1 \%)$ and neutropenia $(9 \%)]$ in $\mathrm{SOLO}^{39}$ or rucaparib treatment [thrombocytopenia (4.5\%) and neutropenia (9.8\%)] in ARIEL2+Study10. ${ }^{42}$ In NOVA, thrombocytopenia was 
transient, platelet levels stabilized after cycle 3 , and treatment discontinuations were not attributed to these hematologic events. ${ }^{45}$ A prominent AE with rucaparib treatment was grade 3/4 alanine aminotransferase (ALT) or aspartate aminotransferase (AST) elevation in ARIEL2+Study 10 $(17 \%)$ or ARIEL3 (10\%); this elevation was transient, self-limiting and not associated with other signs of liver toxicity. $^{42,43}$ Grade 3/4 hypertension occurred in $9 \%$ of niraparib-treated patients and $2 \%$ of patients assigned to placebo. $^{45}$ The most common AEs with veliparib treatment included fatigue, nausea, and vomiting, ${ }^{46,47}$ and AEs with talazoparib treatment included fatigue, anemia and thrombocytopenia. $^{48}$ Other common AEs of any grade observed in PARP inhibitor clinical trials included nausea, vomiting, diarrhea, constipation, fatigue/asthenia, dysgeusia, dyspepsia, decreased appetite, cough, headache, abdominal pain, dyspnea, leukopenia, palpitations, mucositis/ stomatitis, dry mouth, nasopharyngitis, urinary tract infection, myalgia, back pain, arthralgia, dizziness, insomnia, anxiety and rash. Overall, the AEs with PARP inhibitors were managed with appropriate dose reductions and delays. Few patients required dose discontinuation due to serious fatigue or nausea or to other rare treatment-unrelated complications. $^{27,31,42,43,45}$

\section{Combination of PARP inhibitors with chemotherapy or ionizing radiation}

\section{Combination of olaparib with chemotherapy}

Up to $50 \%$ of patients with HGSOC are deficient in the HR pathway for the repair of DNA damage - as a result of germline or somatic BRCA1/2 mutations, epigenetic inactivation of BRCA1, or BRCA-independent defects in the HR pathway. ${ }^{52,53}$ The frequent impairment of $\mathrm{HR}$ repair in HGSOC potentially explains the response to DNA-damaging agents such as platinum compounds. The rationale for combining PARP inhibitors with cytotoxic chemotherapy is the common mechanism of DNA repair and synthetic lethal DNA damage. ${ }^{54,55}$ Carboplatin combined with paclitaxel is the most commonly used chemotherapy regimen to treat patients with platinum-sensitive, recurrent HGSOC. ${ }^{56}$ In a randomized open-label phase II study (NCT01081951), compared with carboplatin and paclitaxel chemotherapy alone, concurrent olaparib with carboplatin and paclitaxel followed by olaparib maintenance significantly improved the PFS of patients with platinum-sensitive, recurrent HGSOC, and the greatest PFS benefit was achieved in patients with BRCA mutations. With regard to OS, the combination schedule did not confer a survival advantage to these patients compared with that of chemotherapy alone. In the combination group, the dose of carboplatin was lower (area under the curve [AUC] $4 \mathrm{mg} / \mathrm{mL}$ per $\mathrm{min}$ ) than that in the chemotherapy group (AUC $6 \mathrm{mg} / \mathrm{mL}$ per min). The dosage of concurrent olaparib was $200 \mathrm{mg}$ twice daily for 10 days; that of sequential olaparib was $400 \mathrm{mg}$ twice daily continuously. The late separation of the PFS curves suggested that the therapeutic benefit was derived mostly from the olaparib maintenance phase. ${ }^{57}$ Increased marrow suppression limits the concurrent use of PARP inhibitors with chemotherapy, and the appropriate dosage of PARP inhibitors to achieve chemopotentiation requires further assessment. Studies will be required to assess the appropriate combination schedules and an effective yet tolerable dosing strategy. Whether the modified concurrent combined with maintenance dosing strategy is more superior than the maintenance alone strategy is still unknown.

\section{Comparison of olaparib with chemotherapy}

The question of whether PARP inhibitors are more effective than cytotoxic chemotherapy for patients with recurrent $\mathrm{EOC}$ and $\mathrm{BRCA} 1 / 2$ mutations is intriguing. In a randomized open-label phase II study (NCT00628251), the efficacy of olaparib (200 $\mathrm{mg}$ or $400 \mathrm{mg}$ twice daily) was compared with that of pegylated liposomal doxorubicin (PLD), which is a DNA-intercalating agent that inhibits topoisomerase II and induces DNA DSBs, in patients with BRCA1/2-mutated recurrent EOC with a less than 12 months platinum-free interval. The PFS time was not significantly different between the $200 \mathrm{mg}$ or $400 \mathrm{mg}$ olaparib and PLD groups, and also between the combined olaparib doses and PLD groups. The median PFS was 6.5, 8.8 , and 7.1 months for the $200 \mathrm{mg}$ olaparib, $400 \mathrm{mg}$ olaparib, and PLD groups, respectively. ${ }^{29}$ In this trial, the median PFS of 7.1 months with PLD therapy was higher than that observed in another phase III randomized trial of patients treated with PLD (PFS of 4 months) with unknown BRCA1/2 status and consistent proportions of platinum-resistant and platinum-sensitive relapsed disease. $^{58} \mathrm{~A}$ retrospective analysis suggested that there might be a potential link between BRCA1/2 mutations and improved clinical benefit with PLD treatment. ${ }^{59}$ Therefore, patients with HRD tumors, including those with BRCA mutations, may derive more benefit from 
chemotherapeutics, including PLD, than unselected patients.

\section{Combination of veliparib with chemotherapy}

Veliparib has been predominantly studied in clinical trials in combination with cytotoxic chemotherapy, probably due to its relatively weak cytotoxicity as a single agent. Currently, trials of veliparib combination strategies are in phase I; the aims of these phase I trials are to investigate the safety, tolerability and preliminary efficacy of veliparib combined with different chemotherapy regimens. Researchers are aiming to investigate the best combination approach to sensitize and potentiate the efficacy of chemotherapy without the cumulative toxicity of chemotherapeutics. In a phase I study (NCT01063816), 54 patients with metastatic or unresectable ovarian cancer treated with $\leq 2$ prior chemotherapy regimens received veliparib combined with carboplatin and gemcitabine, followed by optional veliparib maintenance therapy. Responses were observed in $69 \%$ of patients with BRCA1/2-mutated ovarian cancer (45\% PR, 24\% CR). The most common grade 3/4 AEs and dose-limiting toxicities (DLTs) were neutropenia and thrombocytopenia. The MTD of veliparib was established at $250 \mathrm{mg}$ with carboplatin (AUC 4) plus $800 \mathrm{mg} / \mathrm{m}^{2}$ gemcitabine. Therefore, the combination of veliparib with carboplatin/gemcitabine demonstrated promising preliminary antitumor activity in platinumsensitive ovarian cancer patients with gBRCAm, with a safety profile similar to that of carboplatin and gemcitabine alone. ${ }^{60}$ In another phase I trial, a dose-escalation study (NCT02483104), veliparib combined with carboplatin and weekly paclitaxel, was demonstrated to be tolerated and potentially beneficial for newly diagnosed advanced ovarian cancer, with no DLTs. Grade 3/4 AEs were associated with myelosuppression. The response was assessed in 5 patients $(5 / 9,55.9 \%)$ with measurable disease at baseline; the ORR was $100 \%$ with 4 PRs and $1 \mathrm{CR}$. The recommended phase II dose of veliparib combined with carboplatin/paclitaxel was $150 \mathrm{mg}$ twice daily. ${ }^{61}$

Bevacizumab, which targets vascular endothelial growth factor (VEGF) A, has been approved for concurrent and/or subsequent use in combination with carboplatin and paclitaxel for stage III or IV ovarian cancer following initial surgical resection; in combination with carboplatin and paclitaxel or with carboplatin and gemcitabine for platinum-sensitive recurrent ovarian cancer; and in combination with paclitaxel, PLD, or topotecan for patients with platinum-resistant recurrent ovarian cancer who have received no more than 2 prior chemotherapy regimens. ${ }^{5,62}$ Thus, the MTD and DLTs of veliparib combined with PLD, carboplatin and bevacizumab in recurrent, platinum-sensitive EOC were evaluated in a phase I dose-escalation study (NCT01459380). The DLTs were grade 4 thrombocytopenia and prolonged neutropenia for $>7$ days, grade 3 hypertension, and grade 5 sepsis. Even modest doses of veliparib administered either intermittently or continuously in combination with carboplatin resulted in significant hematologic toxicity in patients with recurrent, platinum-sensitive ovarian cancer. Moreover, the addition of bevacizumab to this regimen carried the risk of additional toxicity associated with VEGF inhibitors. Further exploration of combining PARP inhibitor administration with a lower AUC of carboplatin or administering PARP inhibitors only as maintenance therapy following treatment for platinum-sensitive relapse may be warranted. $^{63}$ In a phase I/II study (NCT01690598), the safety and efficacy of a combination of veliparib and topotecan for the treatment of platinumresistant or partially platinum-sensitive recurrent nongBRCA1/2-mutated EOC were evaluated. However, the best clinical response to this regimen was stable disease. ${ }^{64}$ In another randomized phase II trial (NCT01306032), the response rate of the combination of veliparib with oral cyclophosphamide was compared with that of singleagent oral cyclophosphamide in patients with pretreated BRCA1/2-mutated ovarian cancer. Although the treatment was well tolerated, the addition of veliparib to cyclophosphamide did not improve either the response rate or the median PFS. ${ }^{65}$

\section{Combination of veliparib with ionizing radiation}

In a phase I dose-escalation study (NCT01264432), the efficacy and safety of low-dose fractionated whole abdominal radiation combined with veliparib were investigated in ovarian cancer patients. One $(3.1 \%)$ objective response was observed in a patient with gBRCAm, platinum-sensitive disease. The MTD of veliparib combined with radiation was identified as $250 \mathrm{mg}$ twice daily. The most common grade $3 / 4$ toxicities were fatigue, myelosuppression and gastrointestinal symptoms. ${ }^{66}$ 







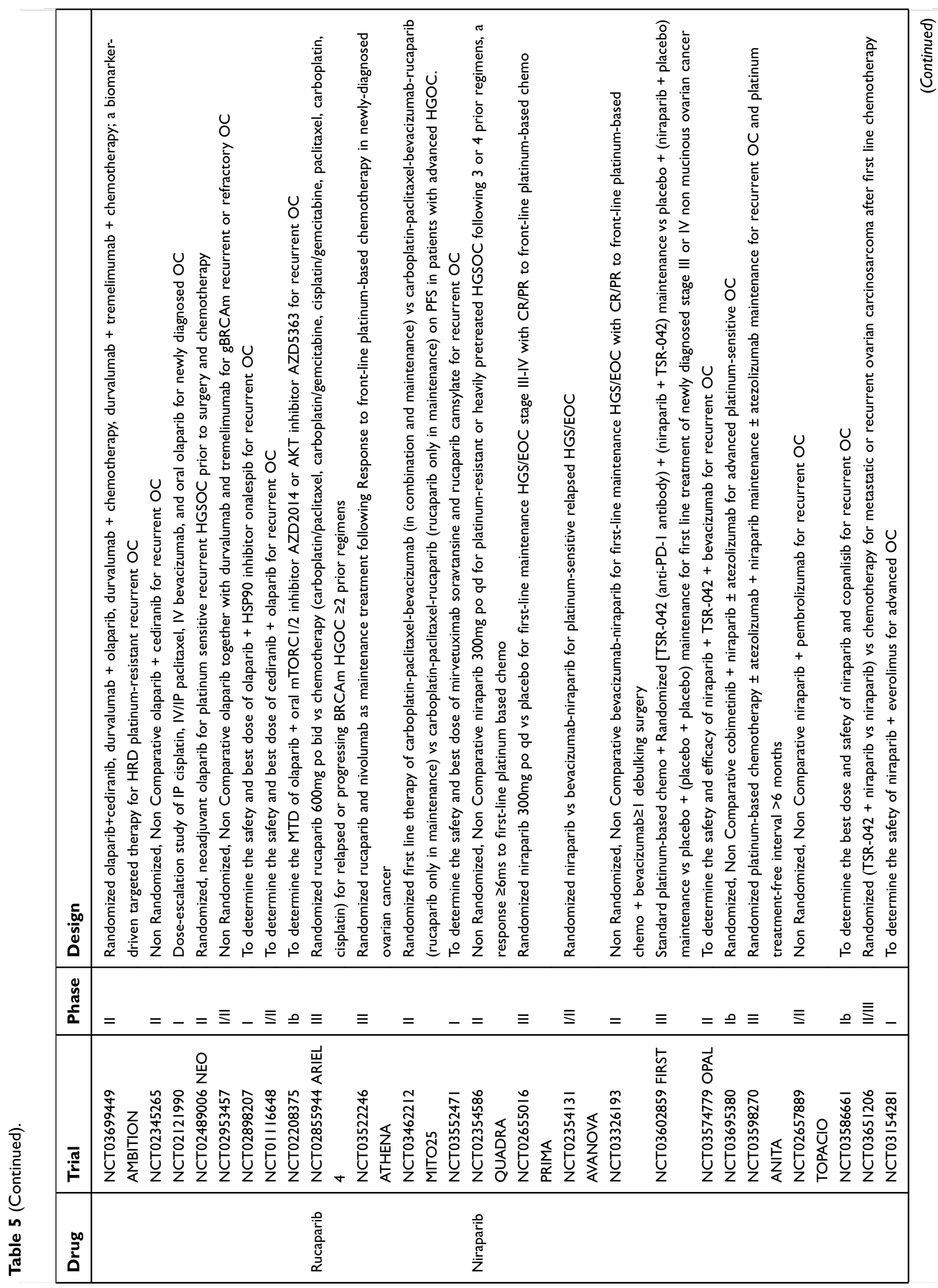




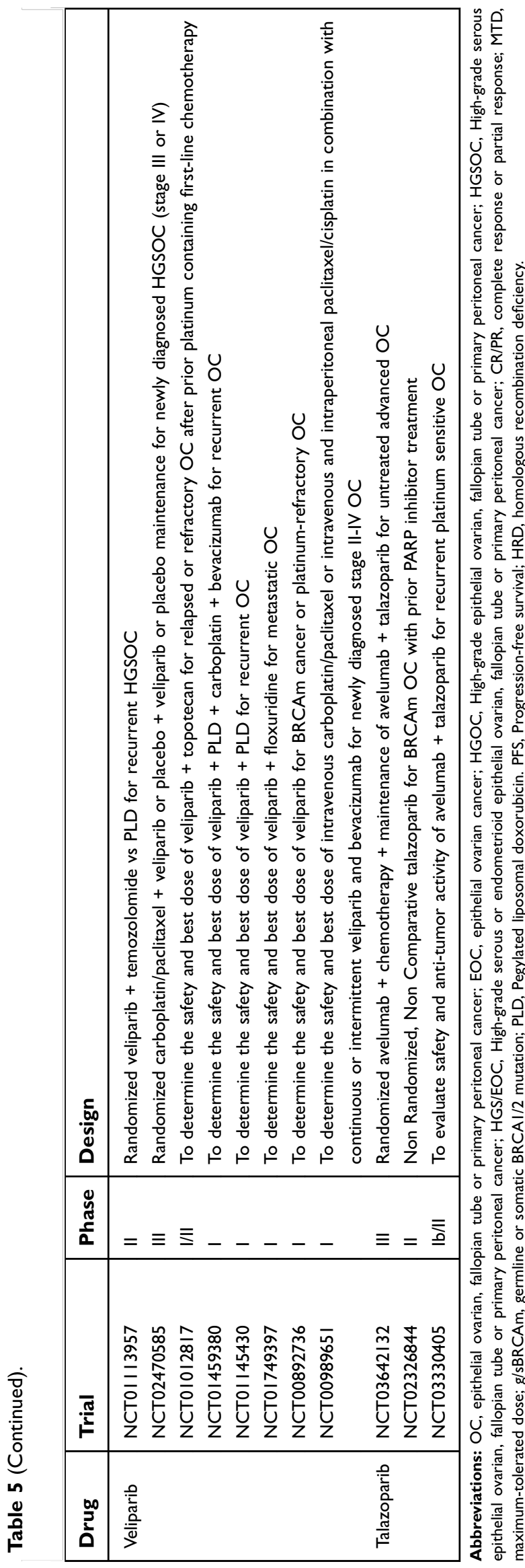

\section{Combination of PARP inhibitors with antiangiogenic agents} Combination of olaparib with antiangiogenic agents

Cediranib is a highly potent inhibitor of VEGF receptors $1-3$. Single-agent cediranib resulted in a response rate of $17 \%$ in a single-arm study of ovarian cancer patients, and the response rate increased to $26 \%$ in platinum-sensitive patients. ${ }^{67,68}$ Cediranib has been shown to cause or augment local tumor hypoxia and has demonstrated an important role in modulating the tumor microenvironment in

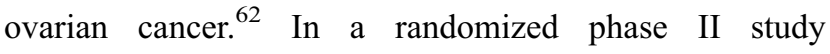
(NCT01116648) comparing single-agent olaparib and combined cediranib/olaparib in patients with platinumsensitive recurrent $\mathrm{HGOC}$, the response rate was high $(79.6 \%)$ in the combination group, with equally notable PFS times of 17.7 months in the combination cohort and 9.0 months in the single-agent olaparib cohort $(p=0.005)$. The gBRCAm status was equally distributed between the combination and single-agent olaparib groups. A PFS time of 5.7 months with single-agent olaparib and 16.5 months ( $p=0.008)$ with combination therapy was observed in the wild-type BRCA group. A PFS time of 16.5 months with single-agent olaparib and 19.4 months $(p=0.16)$ with combination therapy was seen in gBRCAm carriers. Fatigue, diarrhea, and hypertension were the most common grade 3/4 AEs in the combination group, but these AEs were manageable and reversible with supportive care. ${ }^{69}$

\section{Overview of ongoing studies with PARP inhibitors in ovarian cancer}

As of November 2018, 123 clinical trials on PARP inhibitors, including olaparib $(n=59,48.0 \%)$, rucaparib $(n=9$, $7.3 \%)$, niraparib $(n=19,15.4 \%)$, veliparib $(n=26,21.1 \%)$, and talazoparib $(n=10,8.1 \%)$, in ovarian cancer registered in the ClinicalTrials.gov database are ongoing or completed with results yet to be published. Most of these studies focus on combination strategies, including combinations with antiangiogenic agents, chemotherapeutics, and the newly approved bevacizumab. The aims of the combination strategy are to overcome the requirement of BRCA mutation or HRD and to further enhance the efficacy of PARP inhibitors without additional toxicity. Some studies emphasize the role of somatic BRCA mutations, aberrations in other genes in the BRCA pathway or other biomarkers to predict the response to PARP inhibitors. The 
striking improvement in PFS with olaparib maintenance in gBRCA1/2-mutated ovarian cancer patients has not translated into improved OS. Since a substantial proportion of BRCA1/2-mutated HGSOC patients retain sensitivity to platinum following progression on olaparib, it is appropriate to offer a further course of olaparib to these patients. A further course of olaparib might consolidate the gains from the first course of olaparib, improving PFS such that OS also increases. Thus, two or more courses of PARP inhibitor maintenance therapy are initiated, aiming to retain the OS advantage. In addition, more clinical trials are ongoing to investigate the efficacy of PARP inhibitors compared with that of standard-of-care chemotherapy in platinum-resistant or progressive ovarian cancer with or without BRCA mutations. Table 5 shows the critical ongoing trials of PARP inhibitors (olaparib, rucaparib, niraparib, veliparib and talazoparib) in ovarian cancer.

\section{Critical ongoing trials of olaparib in ovarian cancer}

SOLO3 (NCT02282020), a phase III trial of olaparib as maintenance monotherapy, is in progress. ${ }^{31,36}$ SOLO3 aims to confirm whether olaparib is superior to singleagent non-platinum-based chemotherapy (including weekly paclitaxel, topotecan, PLD, or gemcitabine) for patients with gBRCA1/2-mutated platinum-sensitive relapsed ovarian cancer treated with at least two prior platinum-based lines of chemotherapy. Two phase III studies (NCT02446600 and NCT02502266) exploring the combination of cediranib and olaparib in ovarian cancer are ongoing. The first study (NCT02446600) aims to evaluate the efficacy and safety of single-agent olaparib or the combination of cediranib and olaparib versus standard platinum-based chemotherapy to assess the efficacy of these regimens in patients with recurrent platinum-sensitive ovarian cancer. The second study (NCT02502266) aims to evaluate the efficacy and safety of monotherapy with either olaparib or cediranib and the combination of cediranib and olaparib versus non platinum-based standard-of-care chemotherapy in a population of patients with recurrent platinum-resistant or refractory HGSOC enriched for nongBRCAm patients, based on the improved clinical benefit observed with the combination of olaparib and cediranib in patients with BRCA wild-type/unknown status in the phase II study. ${ }^{69}$ The combination strategy might overcome the requirement for BRCA mutation or $\mathrm{HRD}$ and achieve an optimal therapeutic effect. ${ }^{62,69-71}$

\section{Critical ongoing trials of rucaparib in ovarian cancer}

ARIEL2 Part 2 is in progress and will continue to evaluate the HRD status and rucaparib efficacy in ovarian cancer patients treated with at least 3 prior chemotherapy regimens. ARIEL4, a phase III trial (NCT02855944), is underway to further evaluate rucaparib versus standard-of-care chemotherapy regimens, such as carboplatin/paclitaxel, carboplatin/gemcitabine, cisplatin/gemcitabine, or singleagent paclitaxel/carboplatin/cisplatin, in patients with relapsed or progressive, BRCA1/2-mutated HGOC following at least two prior chemotherapy regimens.

\section{Critical ongoing trials of niraparib in ovarian cancer}

QUADRA, a phase II single-arm study, is underway to evaluate the safety and efficacy of niraparib in patients with advanced recurrent HGSOC who have received three or four previous chemotherapy regimens and have previously experienced a response lasting at least 6 months to first-line platinum-based chemotherapy (NCT02354586). QUADRA aims to evaluate the efficacy and safety of niraparib in the setting of 4th-/5th-line treatment for recurrence and the treatment of platinum-resistant or heavily pretreated disease. PRIMA (NCT02655016), a phase III randomized placebo-controlled study, aims to evaluate the efficacy and safety of niraparib maintenance treatment in patients with advanced (FIGO stage III-IV) HGS/EOC who demonstrate clinical CR/PR following the completion of first-line platinum-based chemotherapy. Patients with stage IV disease irrespective of residual disease after primary or interval debulking, inoperable stage III or IV disease, or stage III disease with visible residual disease after primary surgery are eligible for inclusion in this trial. Two trials aiming to evaluate the safety and efficacy of bevacizumab-niraparib combination therapy are ongoing. AVANOVA (NCT02354131) is a two-part, open-label study in which phase I aims to evaluate the safety and tolerability of bevacizumab-niraparib combination therapy, and phase II aims to evaluate the efficacy of niraparib versus bevacizumab-niraparib combination therapy in patients with platinum-sensitive ovarian cancer. Another phase II single-arm study aims to evaluate the safety and efficacy of bevacizumab-niraparib combination therapy as maintenance treatment in patients with advanced ovarian cancer following a response to firstline platinum-based chemotherapy with bevacizumab and 
at least one prior attempt at debulking surgery (NCT03326193).

\section{Critical ongoing trials of veliparib in ovarian cancer}

A phase III clinical trial (NCT02470585) is ongoing to assess the efficacy and safety of veliparib in combination with concurrent and/or subsequent carboplatin/paclitaxel administration for patients with newly diagnosed advanced HGSOC (stage III or IV). In this study, PFS is the primary outcome, and OS and disease-related symptom scores are the secondary outcomes. Four other clinical trials are ongoing to evaluate the role of veliparib in combination with conventional cytotoxic chemotherapy (topotecan, carboplatin/paclitaxel/bevacizumab, PLD, or floxuridine) in first-line and recurrent treatment of ovarian cancer (NCT01012817, NCT00989651, NCT01145430, and NCT01749397).

\section{Critical ongoing trials of talazoparib in ovarian cancer}

A phase II trial (NCT02286687) is in progress to evaluate the efficacy of talazoparib for advanced cancer patients with somatic BRCA mutations, mutations/deletions in PTEN or PTEN loss, HRD, or mutations/deletions in other BRCA pathway genes. In addition, a phase I trial (NCT02316834) is in progress with an aim to determine whether certain characteristics of DNA affect the response of the disease to talazoparib therapy in patients with advanced ovarian cancer that has spread to other anatomical sites and usually cannot be cured or controlled with treatment.

\section{Discussion and conclusion}

Before PARP inhibitors, the only demonstrated efficient strategy for ovarian cancer maintenance treatment was bevacizumab maintenance, which delayed the PFS time to 3-4 months. ${ }^{72-75}$ However, in SOLO2, the PFS was delayed to 15 months, ${ }^{36}$ and in SOLO1, the PFS was delayed to up to 3 years. ${ }^{39}$ Three patients with somatic BRCA1/2-mutated HGSOC were treated for $>5$ years with olaparib monotherapy and reported to achieve durable and long-term responses to olaparib ( $>5$ years), and one of these maintained a response to olaparib for $>7$ years. Notably, at diagnosis, the last patient had a tumor with biallelic somatic deletion and loss-of-function mutation and thereby lacked a functional allele for the recovery of BRCA1 activity, indicating a potential cure. ${ }^{76}$ The safety profiles of PARP inhibitors were mild to moderate, and AEs were manageable and reversible with supportive care. PARP inhibitors confer a certain risk for MDS/AML and should be administered with caution. This risk warrants further investigation.

PARP inhibitor maintenance therapy achieved substantial PFS benefit among patients with platinum-sensitive recurrent ovarian cancer after a $\mathrm{CR} / \mathrm{PR}$ to the most recent regimen. SOLO1 demonstrated that olaparib maintenance therapy following a $\mathrm{CR} / \mathrm{PR}$ to first-line platinum-based chemotherapy confers PFS benefits on patients with advanced primary BRCA1/2-mutated ovarian cancer; moreover, PRIMA, which aims to demonstrate the same indication for niraparib in these patients, is ongoing. Notably, to date, PARP inhibitor clinical trials have focused on serous and endometrioid epithelial ovarian, fallopian tube or primary peritoneal cancers rather than on the mucinous or clear cell subtypes of EOC, carcinosarcoma or undifferentiated ovarian cancer. The significantly improved PFS benefit did not translate into an OS benefit in olaparib trials. The hypothesis that olaparib is insufficiently potent is unlikely to be supported, given the notable HRs in its favor, especially in patients with BRCA mutations. Thus, the most likely explanation for the lack of OS benefit is that effective post-progression therapy overcomes this benefit. Patient crossover to PARP inhibitor treatment after post-progression therapy has been demonstrated to be a confounding factor in analyzing the OS of patients with BRCA mutations. ${ }^{35}$ The effect of PARP inhibitors on prolonging the chemotherapy-free interval might greatly improve patients' quality of life, and studies have shown that resistance to PARP inhibitors does not affect the subsequent response to platinum-based chemotherapy. ${ }^{37,38}$

Clinical trials of chemotherapeutics in combination with PARP inhibitors demonstrated the PFS benefit of the concurrent and sequential combination of olaparib with platinum-based chemotherapy compared with that of platinum-based chemotherapy alone and showed that the benefits are mainly derived from the maintenance monotherapy phase. However, it is unknown whether the regimen of dose-decreasing concurrent chemotherapy with olaparib followed by olaparib maintenance therapy is safer and more efficient than the standard dosing schedule of platinum-based chemotherapy followed by olaparib maintenance therapy for platinum-sensitive recurrent ovarian cancer patients. Considering the increased bone marrow suppression with concurrent use, the optimum strategy 
is sequential rather than concurrent administration. ${ }^{57}$ Some combination strategies expand the utility of PARP inhibitors to HR-proficient tumors. The combination of olaparib and cediranib greatly improved the efficacy of PARP inhibition in gBRCA1/2-mutated ovarian cancers and was also surprisingly active in non-BRCA1/2-mutated ovarian cancers, thus overcoming the requirement for underlying high-level HRD. ${ }^{69}$

To date, three trials have compared the efficacy of PARP inhibitors with that of chemotherapeutic agents in ovarian cancer treatment. PLD, a second-line chemotherapeutic drug, was not significantly different from olaparib in terms of efficacy measured by PFS in patients with BRCA1/2-mutated, recurrent ovarian cancer (disease recurrence within 12 months after prior platinum-based chemotherapy, including platinum-sensitive and platinumresistant disease). ${ }^{29}$ In SOLO3, the efficacy of olaparib is being compared with that of various single-agent nonplatinum chemotherapies for the treatment of BRCA1/2mutated, platinum-sensitive recurrent ovarian cancer. In ARIEL4, the efficacy of rucaparib is being compared with that of standard chemotherapy regimens (including platinum-based chemotherapy) for the treatment of BRCA1/2-mutated, relapsed or progressive ovarian cancer. The latter two trials are ongoing.

Patients with ovarian cancer undergo many relapse and treatment cycles, and the intervals between recurrences shorten with no available chemotherapeutics; moreover, patients die after treatment cessation. Some patients cannot tolerate the toxic effects of chemotherapeutics; thus, drugs cannot be administered at the required time and at the full dose, significantly reducing the effectiveness of chemotherapy, increasing the risk of chemotherapy resistance, and hastening the point at which no further drug is available. With the advent of the era of PARP inhibitors, patients with BRCA1/2-mutated ovarian cancer are treated with PARP inhibitor maintenance therapy following a $\mathrm{CR} /$ PR to platinum-based chemotherapy. As duration of PARP inhibitor maintenance therapy increases, recurrence is expected to be delayed indefinitely. In the near future, BRCA mutation-associated ovarian cancer is expected to become a chronic disease, and an OS benefit will be gained from PARP inhibitor maintenance. This occurrence will be a landmark in ovarian cancer treatment. The death curve for ovarian cancer patients will soon level out. Given the unprecedented PFS benefit of PARP inhibitor maintenance therapy in patients with BRCA1/2-mutated ovarian cancer, clinical trials specifically on PARP inhibitor maintenance therapy for non-BRCA1/2-mutated ovarian cancer should be initiated as soon as possible to augment the understanding of the benefits of PARP inhibitor maintenance therapy and inform the use of PARP inhibitors in this patient population.

\section{Abbreviation list}

PARP, Poly (ADP-ribose) polymerase; BRCA, Breast Cancer Susceptibility Gene; FDA, Food and Drug Administration; EMA, European Medicine Agency; EOC, Epithelial ovarian cancer; PFS, progression-free survival; OC, ovarian cancer; HRD, homologous recombination-deficient; EU, Europe; US, the United States; SSBs, single-strand breaks; DSBs, double-strand breaks; HR, homologous recombination; NHEJ, nonhomologous endjoining; g/s, germline and/or somatic; HGSOC, high-grade serous epithelial ovarian, fallopian tube or primary peritoneal cancer; $\mathrm{CR} / \mathrm{PR}$, complete or partial response; gBRCAm, germline BRCA mutation; HGOC, high-grade ovarian carcinoma; ORR, objective response rate; OS, overall survival; MTD, maximum tolerated dose; HR, hazard ratio; 95\% CI, 95\% confidence interval; HGS/ EOC, high-grade serous or endometrioid ovarian cancer, primary peritoneal cancer or fallopian tube cancer; $\mathrm{LOH}$, loss of heterozygosity; AEs, adverse events; MDS/AML, myelodysplastic syndrome/acute myeloid leukemia; ALT, alanine aminotransferase; AST, aspartate aminotransferase; AUC, area under the curve; PLD, pegylated liposomal doxorubicin; DLTs, dose-limiting toxicities; VEGF, vascular endothelial growth factor.

\section{Acknowledgments}

This study was supported by the National Natural Science Foundation of China (81702551) and the Capital Health Research and Development of Special Funding (Project No. 2018-1-1061).

\section{Disclosure}

The authors report no conflicts of interest in this work.

\section{References}

1. Siegel RL, Miller KD, Jemal A. Cancer statistics, 2017. CA Cancer J Clin. 2017;67:7-30. doi:10.3322/caac.21387

2. Jayson GC, Kohn EC, Kitchener HC, Ledermann JA. Ovarian cancer. Lancet. 2014;384:1376-1388. doi:10.1016/S0140-6736(13)62146-7

3. Heintz APM, Odicino F, Maisonneuve P, et al. Carcinoma of the ovary. Int J Gynecology Obstetrics. 2006;95:S161-S92. doi:10.1016/S00207292(06)60033-7 
4. Hanker LC, Loibl S, Burchardi N, et al. The impact of second to sixth line therapy on survival of relapsed ovarian cancer after primary taxane/platinum-based therapy. Ann Oncol. 2012;23:2605-2612. doi:10.1093/annonc/mds203

5. Genentech, Inc. Prescribing information for Avastin (bevacizumab) injection, for intravenous use.Available from: https:/www.access data.fda.gov/drugsatfda_docs/label/2018/125085s323lbl.pdf. Accessed June 13, 2018.

6. Liu JF, Konstantinopoulos PA, Matulonis UA. PARP inhibitors in ovarian cancer: current status and future promise. Gynecol Oncol. 2014;133:362-369. doi:10.1016/j.ygyno.2014.02.039

7. Deeks ED. Olaparib: first global approval. Drugs. 2015;75:231-240. doi:10.1007/s40265-015-0345-6

8. Scott LJ. Niraparib: first global approval. Drugs. 2017;77:10291034. doi:10.1007/s40265-017-0752-y

9. Syed YY. Rucaparib: first global approval. Drugs. 2017;77:585-592. doi:10.1007/s40265-017-0716-2

10. Lord CJ, Ashworth A. The DNA damage response and cancer therapy. Nature. 2012;481:287-294. doi:10.1038/nature10760

11. Jones P, Wilcoxen K, Rowley M, Toniatti C. Niraparib: a poly(ADPribose) polymerase (PARP) inhibitor for the treatment of tumors with defective homologous recombination. J Med Chem. 2015;58:33023314. doi:10.1021/jm5018237

12. Lord CJ, Ashworth A. Mechanisms of resistance to therapies targeting BRCA-mutant cancers. Nat Med. 2013;19:1381-1388. doi:10.1038/ nm.3369

13. Plummer R. Perspective on the pipeline of drugs being developed with modulation of DNA damage as a target. Clin Cancer Res. 2010;16:4527-4531. doi:10.1158/1078-0432.CCR-10-0984

14. Bryant HE, Schultz N, Thomas HD, et al. Specific killing of BRCA2deficient tumours with inhibitors of poly(ADP-ribose) polymerase. Nature. 2005;434:913-917. doi:10.1038/nature03443

15. Farmer H, McCabe N, Lord CJ, et al. Targeting the DNA repair defect in BRCA mutant cells as a therapeutic strategy. Nature. 2005;434:917-921. doi:10.1038/nature03445

16. Murai J, Huang SY, Das BB, et al. Trapping of PARP1 and PARP2 by clinical PARP inhibitors. Cancer Res. 2012;72:5588-5599. doi:10.1158/0008-5472.CAN-12-2753

17. AstraZeneca AB. Lynparza $50 \mathrm{mg}$ hard capsules: EU summary of product characteristics; 2014. Available from: https://www.ema. europa.eu/documents/product-information/lynparza-epar-productinformation_en.pdf. Accessed January 9, 2015.

18. AstraZeneca Pharmaceuticals LP. Lynparza (olaparib) capsules, for oral use: US prescribing information; 2014. Available from: https:// www.accessdata.fda.gov/drugsatfda_docs/label/2018/206162s011lbl. pdf. Accessed January 5, 2015.

19. AstraZeneca Pharmaceuticals LP. Lynparza (olaparib) tablets, for oral use: FDA approved olaparib tablets for maintenance treatment in ovarian cancer. Available from: https:/www.accessdata.fda.gov/drugsatfda docs/label/2018/208558s005lbl.pdf. Accessed September 26, 2018.

20. AstraZeneca AB. Lynparza $100 \mathrm{mg}$ and $150 \mathrm{mg}$ tablet: EU CHMP post-authorisation summary of positive opinion for lynparza. 2018. Available from: https:/www.ema.europa.eu/documents/smop/chmppost-authorisation-summary-positive-opinion-lynparza_en.pdf. Accessed February 23, 2018.

21. AstraZeneca Pharmaceuticals LP. Lynparza (olaparib) tablets, for oral use: US prescribing information; 2018. Available from: https://www. accessdata.fda.gov/drugsatfda_docs/label/2018/208558s006lbl.pdf. Accessed December 19, 2018.

22. Clovis Oncology Inc. Prescribing information for rubraca (rucaparib) tablets, for oral use. 2016. Available from: https://www.accessdata. fda.gov/drugsatfda_docs/label/2018/209115s0031bl.pdf. Accessed April 6, 2018.

23. Clovis Oncology Inc. Rubraca tablet: EU CHMP summary of positive opinion for rubraca. 2018. Available from: https://www.ema.europa.eu/ en/medicines/human/EPAR/rubraca. Accessed March 23, 2018.
24. Tesaro. ZEJULA(niraparib): US prescribing information. 2017. Available from: https://www.accessdata.fda.gov/drugsatfda_docs/ label/2017/2084471bl.pdf. Accessed March 29, 2017.

25. Tesaro. Zejula $100 \mathrm{mg}$ hard capsules: EU summary of product characteristics. 2017. Available from: https://www.ema.europa.eu/docu ments/product-information/zejula-epar-product-information_en.pdf. Accessed November, 272017.

26. Kaufman B, Shapira-Frommer R, Schmutzler RK, et al. Olaparib monotherapy in patients with advanced cancer and a germline BRCA1/2 mutation. J Clin Oncol. 2015;33:244-250. doi:10.1200/ JCO.2014.56.2728

27. Matulonis UA, Penson RT, Domchek SM, et al. Olaparib monotherapy in patients with advanced relapsed ovarian cancer and a germline BRCA1/2 mutation: a multistudy analysis of response rates and safety. Ann Oncol. 2016;27:1013-1019. doi:10.1093/ annonc/mdw133

28. Fong PC, Boss DS, Yap TA, et al. Inhibition of poly(ADP-ribose) polymerase in tumors from BRCA mutation carriers. $N$ Engl J Med. 2009;361:123-134. doi:10.1056/NEJMoa0900212

29. Kaye SB, Lubinski J, Matulonis U, et al. Phase II, open-label, randomized, multicenter study comparing the efficacy and safety of olaparib, a poly (ADP-ribose) polymerase inhibitor, and pegylated liposomal doxorubicin in patients with BRCA1 or BRCA2 mutations and recurrent ovarian cancer. J Clin Oncol. 2012;30:372-379. doi:10.1200/JCO.2011.36.9215

30. Audeh MW, Carmichael J, Penson RT, et al. Oral poly(ADP-ribose) polymerase inhibitor olaparib in patients with BRCA1 or BRCA2 mutations and recurrent ovarian cancer: a proof-of-concept trial. Lancet. 2010;376:245-251. doi:10.1016/S0140-6736(10)60893-8

31. Ledermann J, Harter P, Gourley C, et al. Olaparib maintenance therapy in platinum-sensitive relapsed ovarian cancer. $N$ Engl J Med. 2012;366:1382-1392. doi:10.1056/NEJMoa1105535

32. Ledermann J, Harter P, Gourley C, et al. Olaparib maintenance therapy in patients with platinum-sensitive relapsed serous ovarian cancer: a preplanned retrospective analysis of outcomes by BRCA status in a randomised phase 2 trial. Lancet Oncol. 2014;15:852-861. doi:10.1016/S1470-2045(14)70228-1

33. Ledermann JA, Harter P, Gourley C, et al. Overall survival in patients with platinum-sensitive recurrent serous ovarian cancer receiving olaparib maintenance monotherapy: an updated analysis from a randomised, placebo-controlled, double-blind, phase 2 trial. Lancet Oncol. 2016;17:1579-1589. doi:10.1016/S1470-2045(16)30376-X

34. Friedlander M, Matulonis U, Gourley C, et al. Long-term efficacy, tolerability and overall survival in patients with platinum-sensitive, recurrent high-grade serous ovarian cancer treated with maintenance olaparib capsules following response to chemotherapy. Br J Cancer. 2018;119:1075-1085. doi:10.1038/s41416-018-0271-y

35. Matulonis UA, Harter P, Gourley C, et al. Olaparib maintenance therapy in patients with platinum-sensitive, relapsed serous ovarian cancer and a BRCA mutation: overall survival adjusted for postprogression poly(adenosine diphosphate ribose) polymerase inhibitor therapy. Cancer. 2016;122:1844-1852. doi:10.1002/cncr.29995

36. Pujade-Lauraine E, Ledermann JA, Selle F, et al. Olaparib tablets as maintenance therapy in patients with platinum-sensitive, relapsed ovarian cancer and a BRCA1/2 mutation (SOLO2/ ENGOT-Ov21): a double-blind, randomised, placebo-controlled, phase 3 trial. Lancet Oncol. 2017;18:1274-1284. doi:10.1016/ S1470-2045(17)30469-2

37. Konstantinopoulos PA, Ceccaldi R, Shapiro GI, D‘Andrea AD. Homologous recombination deficiency: exploiting the fundamental vulnerability of ovarian cancer. Cancer Discov. 2015;5:1137-1154. doi:10.1158/2159-8290.CD-15-0714

38. Ang JE, Gourley C, Powell CB, et al. Efficacy of chemotherapy in BRCA1/2 mutation carrier ovarian cancer in the setting of PARP inhibitor resistance: a multi-institutional study. Clin Cancer Res. 2013;19:5485-5493. doi:10.1158/1078-0432.CCR-13-1262 
39. Moore K, Colombo N, Scambia G, et al. Maintenance olaparib in patients with newly diagnosed advanced ovarian cancer. $N$ Engl $J$ Med. 2018. doi:10.1056/NEJMoa1810858

40. Kristeleit R, Shapiro GI, Burris HA, et al. A phase I-II study of the oral PARP inhibitor rucaparib in patients with germline BRCA1/2mutated ovarian carcinoma or other solid tumors. Clin Cancer Res. 2017;23:4095-4106. doi:10.1158/1078-0432.CCR-16-2796

41. Swisher EM, Lin KK, Oza AM, et al. Rucaparib in relapsed, platinum-sensitive high-grade ovarian carcinoma (ARIEL2 part 1): an international, multicentre, open-label, phase 2 trial. Lancet Oncol. 2017;18:75-87. doi:10.1016/S1470-2045(16)30559-9

42. Oza AM, Tinker AV, Oaknin A, et al. Antitumor activity and safety of the PARP inhibitor rucaparib in patients with high-grade ovarian carcinoma and a germline or somatic BRCA1 or BRCA2 mutation: integrated analysis of data from study 10 and ARIEL2. Gynecol Oncol. 2017;147:267-275. doi:10.1016/j.ygyno.2017.08.022

43. Coleman RL, Oza AM, Lorusso D, et al. Rucaparib maintenance treatment for recurrent ovarian carcinoma after response to platinum therapy (ARIEL3): a randomised, double-blind, placebo-controlled, phase 3 trial. Lancet. 2017;390:1949-1961. doi:10.1016/S0140-6736(17)32440-6

44. Sandhu SK, Schelman WR, Wilding G, et al. The poly(ADP-ribose) polymerase inhibitor niraparib (MK4827) in BRCA mutation carriers and patients with sporadic cancer: a phase 1 dose-escalation trial. Lancet Oncol. 2013;14:882-892. doi:10.1016/S1470-2045(13)70240-7

45. Mirza MR, Monk BJ, Herrstedt J, et al. Niraparib maintenance therapy in platinum-sensitive, recurrent ovarian cancer. $N$ Engl J Med. 2016;375:2154-2164. doi:10.1056/NEJMoa1611310

46. Steffensen KD, Adimi P, Jakobsen A. Veliparib monotherapy to patients with BRCA germ line mutation and platinum-resistant or partially platinum-sensitive relapse of epithelial ovarian cancer: a phase I/II study. Int J Gynecol Cancer. 2017;27:1842-1849. doi:10.1097/IGC.0000000000001089

47. Coleman RL, Sill MW, Bell-McGuinn K, et al. A phase II evaluation of the potent, highly selective PARP inhibitor veliparib in the treatment of persistent or recurrent epithelial ovarian, fallopian tube, or primary peritoneal cancer in patients who carry a germline BRCA1 or BRCA2 mutation - an NRG oncology/gynecologic oncology group study. Gynecol Oncol. 2015;137:386-391. doi:10.1016/j.ygyno.2015.03.042

48. de Bono J, Ramanathan RK, Mina L, et al. Phase I, dose-escalation, two-part trial of the PARP inhibitor talazoparib in patients with advanced germline BRCA1/2 mutations and selected sporadic cancers. Cancer Discov. 2017;7:620-629. doi:10.1158/2159-8290.CD-16-1250

49. Travis LB, Holowaty EJ, Bergfeldt K, et al. Risk of leukemia after platinum-based chemotherapy for ovarian cancer. $N \mathrm{Engl} \mathrm{J} \mathrm{Med.}$ 1999;340:351-357. doi:10.1056/NEJM199902043400504

50. Vay A, Kumar S, Seward S, et al. Therapy-related myeloid leukemia after treatment for epithelial ovarian carcinoma: an epidemiological analysis. Gynecol Oncol. 2011;123:456-460. doi:10.1016/j. ygyno.2011.07.097

51. Shenolikar R, Durden E, Meyer N, Lenhart G, Moore K. Incidence of secondary myelodysplastic syndrome (MDS) and acute myeloid leukemia (AML) in patients with ovarian or breast cancer in a real-world setting in the United States. Gynecol Oncol. 2018;151:190-195. doi:10.1016/j.ygyno.2018.09.003

52. Press JZ, De Luca A, Boyd N, et al. Ovarian carcinomas with genetic and epigenetic BRCA1 loss have distinct molecular abnormalities. BMC Cancer. 2008;8:17. doi:10.1186/1471-2407-8-172

53. Cancer Genome Atlas Research N. Integrated genomic analyses of ovarian carcinoma. Nature. 2011;474:609-615. doi:10.1038/nature10166

54. Evers B, Drost R, Schut E, et al. Selective inhibition of BRCA2-deficient mammary tumor cell growth by AZD2281 and cisplatin. Clin Cancer Res. 2008;14:3916-3925. doi:10.1158/1078-0432.CCR-07-4953

55. Rottenberg S, Jaspers JE, Kersbergen A, et al. High sensitivity of BRCA1-deficient mammary tumors to the PARP inhibitor AZD2281 alone and in combination with platinum drugs. Proc Natl Acad Sci U $S$ A. 2008;105:17079-17084. doi:10.1073/pnas.0806092105
56. Parmar MK, Ledermann JA, Colombo N, et al. Paclitaxel plus platinum-based chemotherapy versus conventional platinum-based chemotherapy in women with relapsed ovarian cancer: the ICON4/ AGO-OVAR-2.2 trial. Lancet. 2003;361:2099-2106.

57. Oza AM, Cibula D, Benzaquen AO, et al. Olaparib combined with chemotherapy for recurrent platinum-sensitive ovarian cancer: a randomised phase 2 trial. Lancet Oncol. 2015;16:87-97. doi:10.1016/ S1470-2045(14)71135-0

58. Gordon AN, Fleagle JT, Guthrie D, Parkin DE, Gore ME, Lacave AJ. Recurrent epithelial ovarian carcinoma: a randomized phase III study of pegylated liposomal doxorubicin versus topotecan. J Clin Oncol. 2001;19:3312-3322. doi:10.1200/JCO.2001.19.14.3312

59. Safra T, Borgato L, Nicoletto MO, et al. BRCA mutation status and determinant of outcome in women with recurrent epithelial ovarian cancer treated with pegylated liposomal doxorubicin. $\mathrm{Mol}$ Cancer Ther. 2011;10:2000-2007. doi:10.1158/1535-7163.MCT$11-0272$

60. Gray HJ, Bell-McGuinn K, Fleming GF, et al. Phase I combination study of the PARP inhibitor veliparib plus carboplatin and gemcitabine in patients with advanced ovarian cancer and other solid malignancies. Gynecol Oncol. 2018;148:507-514. doi:10.1016/j. ygyno.2017.12.029

61. Nishio S, Takekuma M, Takeuchi S, et al. Phase 1 study of veliparib with carboplatin and weekly paclitaxel in Japanese patients with newly diagnosed ovarian cancer. Cancer Sci. 2017;108:2213-2220. doi:10.1111/cas.13381

62. Ivy SP, Liu JF, Lee JM, Matulonis UA, Kohn EC. Cediranib, a pan-VEGFR inhibitor, and olaparib, a PARP inhibitor, in combination therapy for high grade serous ovarian cancer. Expert Opin Investig Drugs. 2016;25:597-611. doi:10.1517/ 13543784.2016.1156857

63. Landrum LM, Brady WE, Armstrong DK, et al. A phase I trial of pegylated liposomal doxorubicin (PLD), carboplatin, bevacizumab and veliparib in recurrent, platinum-sensitive ovarian, primary peritoneal, and fallopian tube cancer: an NRG oncology/gynecologic oncology group study. Gynecol Oncol. 2016;140:204-209. doi:10.1016/j.ygyno.2015.11.024

64. Hjortkjaer M, Kanstrup H, Jakobsen A, Steffensen KD. Veliparib and topotecan for patients with platinum-resistant or partially platinumsensitive relapse of epithelial ovarian cancer with BRCA negative or unknown BRCA status. Cancer Treat Res Commun. 2018;14:7-12. doi:10.1016/j.ctarc.2017.09.001

65. Kummar S, Oza AM, Fleming GF, et al. Randomized trial of oral cyclophosphamide and veliparib in high-grade serous ovarian, primary peritoneal, or fallopian tube cancers, or brca-mutant ovarian cancer. Clin Cancer Res. 2015;21:1574-1582. doi:10.1158/10780432.CCR-14-2565

66. Reiss KA, Herman JM, Armstrong D, et al. A final report of a phase I study of veliparib (ABT-888) in combination with low-dose fractionated whole abdominal radiation therapy (LDFWAR) in patients with advanced solid malignancies and peritoneal carcinomatosis with a dose escalation in ovarian and fallopian tube cancers. Gynecol Oncol. 2017;144:486-490. doi:10.1016/j.ygyno.2017.01.016

67. Hirte H, Lheureux S, Fleming GF, et al. A phase 2 study of cediranib in recurrent or persistent ovarian, peritoneal or fallopian tube cancer: a trial of the princess margaret, chicago and california phase II consortia. Gynecol Oncol. 2015;138:55-61. doi:10.1016/j. ygyno.2015.04.009

68. Matulonis UA, Berlin S, Ivy P, et al. Cediranib, an oral inhibitor of vascular endothelial growth factor receptor kinases, is an active drug in recurrent epithelial ovarian, fallopian tube, and peritoneal cancer. $J$ Clin Oncol. 2009;27:5601-5606. doi:10.1200/JCO.2009.23.2777

69. Liu JF, Barry WT, Birrer M, et al. Combination cediranib and olaparib versus olaparib alone for women with recurrent platinumsensitive ovarian cancer: a randomised phase 2 study. Lancet Oncol. 2014;15:1207-1214. doi:10.1016/S1470-2045(14)70391-2 
70. Liu JF, Tolaney SM, Birrer M, et al. 1 trial of the poly(ADP-ribose) polymerase inhibitor olaparib (AZD2281) in combination with the anti-angiogenic cediranib (AZD2171) in recurrent epithelial ovarian or triple-negative breast cancer. Eur J Cancer. 2013;49:2972-2978. doi:10.1016/j.ejca.2013.05.020

71. Lu Y, Chu A, Turker MS, Glazer PM. Hypoxia-induced epigenetic regulation and silencing of the BRCA1 promoter. Mol Cell Biol. 2011;31:3339-3350. doi:10.1128/MCB.01121-10

72. Perren TJ, Swart AM, Pfisterer J, et al. A phase 3 trial of bevacizumab in ovarian cancer. $N$ Engl J Med. 2011;365:2484-2496. doi:10.1056/ NEJMoa1103799

73. Burger RA, Brady MF, Bookman MA, et al. Incorporation of bevacizumab in the primary treatment of ovarian cancer. NEngl J Med. 2011;365:24732483. doi:10.1056/NEJMoa1104390
74. Pujade-Lauraine E, Hilpert F, Weber B, et al. Bevacizumab combined with chemotherapy for platinum-resistant recurrent ovarian cancer: the AURELIA open-label randomized phase III trial. $J$ Clin Oncol. 2014;32:1302-1308. doi:10.1200/JCO.2013.51.44 89

75. Aghajanian C, Blank SV, Goff BA, et al. OCEANS: a randomized, double-blind, placebo-controlled phase III trial of chemotherapy with or without bevacizumab in patients with platinum-sensitive recurrent epithelial ovarian, primary peritoneal, or fallopian tube cancer. J Clin Oncol. 2012;30:2039-2045. doi:10.1200/JCO.2012.42.0505

76. Lheureux S, Bruce JP, Burnier JV, et al. Somatic BRCA1/2 recovery as a resistance mechanism after exceptional response to poly (ADPribose) polymerase inhibition. J Clin Oncol. 2017;35:1240-1249. doi: $10.1200 /$ JCO.2016.71.3677

\section{Publish your work in this journal}

Cancer Management and Research is an international, peer-reviewed open access journal focusing on cancer research and the optimal use of preventative and integrated treatment interventions to achieve improved outcomes, enhanced survival and quality of life for the cancer patient.
The manuscript management system is completely online and includes a very quick and fair peer-review system, which is all easy to use. Visit http://www.dovepress.com/testimonials.php to read real quotes from published authors. 